\title{
Differences between asthma-COPD overlap syndrome and adult-onset asthma
}

\author{
Minna Tommola ${ }^{1}$, Pinja Ilmarinen ${ }^{1}$, Leena E. Tuomisto ${ }^{1}$, Lauri Lehtimäki ${ }^{2,3}$, \\ Jussi Haanpää4 , Onni Niemelä $\ddot{3}^{3,5}$ and Hannu Kankaanrantata ${ }^{1,3}$ \\ Affiliations: ${ }^{1}$ Dept of Respiratory Medicine, Seinäjoki Central Hospital, Seinäjoki, Finland. ${ }^{2}$ Allergy Centre, \\ Tampere University Hospital, Tampere, Finland. ${ }^{3}$ Faculty of Medicine and Life Sciences, University of \\ Tampere, Tampere, Finland. " $D$ Dept of Clinical Physiology, Seinäjoki Central Hospital, Seinäjoki, Finland. \\ ${ }^{5}$ Dept of Laboratory Medicine and Medical Research Unit, Seinäjoki Central Hospital, Seinäjoki, Finland.
}

Correspondence: Minna Tommola, Dept of Respiratory Medicine, Seinäjoki Central Hospital, FIN-60220 Seinäjoki, Finland. E-mail: minna.tommoladepshp.fi

@ERSpublications

ACOS differs from adult-onset asthma, with lower diffusing capacity, and higher serum IL-6 and blood neutrophil count http://ow.ly/Uxa030aqJAR

Cite this article as: Tommola M, Ilmarinen P, Tuomisto LE, et al. Differences between asthma-COPD overlap syndrome and adult-onset asthma. Eur Respir J 2017; 49: 1602383 [https://doi.org/10.1183/ 13993003.02383-2016].

ABSTRACT Differences between asthma-COPD overlap syndrome (ACOS) and adult-onset asthma are poorly understood. This study aimed to evaluate these differences in a clinical cohort of patients with adult-onset asthma, as a part of the Seinäjoki Adult Asthma Study (SAAS).

188 patients were diagnosed with adult-onset asthma and re-evaluated 12 years after diagnosis. They were divided into three groups based on smoking history and post bronchodilator spirometry values: 1) never- and ex-smokers with $<10$ smoked pack-years; 2) non-obstructive (forced expiratory volume in $1 \mathrm{~s}$ $(\mathrm{FEV} 1) /$ forced vital capacity $(\mathrm{FVC}) \geqslant 0.7)$ patients with $\geqslant 10$ pack-years; and 3 ) ACOS patients with $\geqslant 10$ pack-years and $\mathrm{FEV} 1 / \mathrm{FVC}<0.7$.

ACOS patients had lower diffusing capacity (DLCO/VA $86 \%$ predicted versus 98 or $96 \%$ predicted; $\mathrm{p}<0.001$ ), higher blood neutrophil levels (4.50 versus 3.60 or $3.85 \times 10^{9} \mathrm{~L}^{-1} ; \mathrm{p}=0.008$ ), and higher IL-6 levels ( 2.88 versus 1.52 or $2.10 \mathrm{pg} \cdot \mathrm{mL}^{-1}, \mathrm{p}<0.001$ ) as compared to never- and ex-smokers with $<10$ pack-years, or non-obstructive patients with $\geqslant 10$ pack-years smoking history, respectively. ACOS patients also showed reduced lung function, higher remaining bronchial reversibility and a higher number of comorbidities.

This study shows distinct differences in diffusing capacity, blood neutrophil and IL-6 levels, bronchial reversibility, lung function and comorbidities between ACOS and adult-onset asthma. The present findings should be considered in the comprehensive assessment of adult asthma patients.

This article has supplementary material available from erj.ersjournals.com

Received: Dec 052016 | Accepted after revision: Feb 252017

This study is registered at ClinicalTrials.gov with identifier number NCT02733016.

Support statement: Supported by the Finnish Anti-Tuberculosis Association Foundation (Helsinki, Finland), Tampere Tuberculosis Foundation (Tampere, Finland), Jalmari and Rauha Ahokas Foundation (Helsinki, Finland), the Research Foundation of the Pulmonary Diseases (Helsinki, Finland), the Competitive State Research Financing of the Expert Responsibility Area of Tampere University Hospital (Tampere, Finland) and the Medical Research Fund of Seinäjoki Central Hospital (Seinäjoki, Finland). None of the sponsors was involved in the planning, execution, drafting or write-up of this study. Funding information for this article has been deposited with the Crossref Funder Registry.

Conflict of interest: Disclosures can be found alongside this article at erj.ersjournals.com

Copyright @ERS 2017 


\section{Introduction}

Asthma and chronic obstructive pulmonary disease (COPD) have previously been categorised as separate entities of obstructive airway disease with different clinical features [1,2]. Recently, however, overlapping of these two diseases has been recognised, and a novel clinical phenotype, asthma-COPD overlap syndrome (ACOS) has been described. ACOS is characterised by persistent airway obstruction accompanied by several features of both asthma and COPD [3-6]. ACOS has recently been recognised by the Global Initiative for Asthma (GINA) and Global Initiative for Chronic Obstructive Lung Disease (GOLD), and has been included in several national guidelines of COPD $[3-5,7]$. ACOS is considered to develop mainly by two different pathways: either the patient with COPD develops asthma-like symptoms and/or typical characteristics of asthma (for example, high reversibility of the airways); or the patient with asthma continues to smoke and eventually develops non-reversible airway obstruction indicating COPD [8, 9]. There is also a third pathway suggested, in which a patient with asthma develops non-reversible airway obstruction without a smoking history [9]. However, a history of exposure to tobacco smoking (or biomass fuels) has been considered a requirement for COPD diagnosis [2]. Thus, it has been proposed that smoking is also to be regarded as a necessary factor when using the asthma-COPD overlap diagnosis $[8,10]$.

Previous studies on asthma have generally excluded smoking patients, and studies of COPD have mostly excluded patients with a history or diagnosis of asthma. Therefore, relatively little is known about the differences between ACOS and asthma $[6,10]$. The prevalence of ACOS among patients with COPD or asthma is suggested to be $12 \%-61 \%$ depending on the criteria used [9], and it is reported to increase with age [11]. Previous studies on ACOS have been conducted in COPD cohorts mainly, and ACOS patients are reported to have more frequent exacerbations [12-14] and hospitalisations [11, 14], poorer quality of life [12], reduced physical activity [12] and increased dyspnoea and wheezing, as compared to patients with COPD alone $[11,12]$.

ACOS among asthmatic patients, remains far less frequently studied. Some epidemiological and registry-based studies on ACOS among patients with asthma have been previously published, leaving a major need for clinical studies with actual patients. However, these previous studies have reported more frequent exacerbations [14, 15], poorer asthma control, increased symptoms of dyspnoea [15], impaired lung function [14-16] and poorer quality of life [16] in ACOS patients, as compared to patients with asthma alone. Patients with ACOS are also reported to have an increased rate $[14,17]$ and duration of hospitalisation, as compared to patients with asthma [17]. Furthermore, the number of comorbidities [15], particularly hypertension $[15,16]$, has been reported to be higher among patients with ACOS as compared to those with asthma alone, and ACOS patient mortality has been suggested to be higher than that of patients with asthma $[18,19]$.

Diagnosis of ACOS is challenging, because no specific single clinical feature, spirometric finding, or biomarker has been identified to differentiate ACOS from asthma [6, 20,21]. Differentiating ACOS from COPD has been considered important, as it affects the choice of therapy, i.e. the use of inhaled glucocorticoids. Differentiating ACOS from asthma, however, has received less attention, even though there are options of targeted treatment for COPD, such as long-acting muscarinic antagonists (LAMA) and roflumilast. Furthermore, ACOS is presently a phenotype with heterogenic and poorly defined clinical features. For this reason, there is an urgent need for the identification of specific characteristics and biomarkers of ACOS [20].

The aim of this study was to evaluate the differences between asthma and ACOS in a clinical cohort of patients with adult-onset asthma.

\section{Methods}

Study population and design

The Seinäjoki Adult Asthma Study (SAAS) was a 12-year follow-up study (during the years 1999-2013), in which 257 patients were diagnosed with adult-onset asthma (asthma onset at the age of $\geqslant 15$ years) in the Dept of Respiratory Medicine of Seinäjoki Central Hospital, Finland. Diagnosis of asthma was made by a respiratory physician, based on typical symptoms and was confirmed by objective lung function measurements. The study protocol and inclusion and exclusion criteria have been previously published (table S1) [22]. Smokers (current or ex-) were included. After a follow-up of 12 years, 203 patients (79\%) were re-evaluated (years 2012-2013), and the data of 188 patients were included in the analysis (figure 1). During the follow-up, patients were actively treated for asthma, according to the Finnish Asthma Programme guidelines [23]. Medication use was recorded, using a structured questionnaire that included self-reported medication at the time of the follow-up visit. The present study was cross-sectional, using data mostly from the control visit (years 2012-2013). However, when assessing the use of oral corticosteroid courses, atopy or airway obstruction at baseline, longitudinal data was utilised. Written informed consent was obtained from participants and the study protocol was approved by the Ethics Committee of Tampere University Hospital, Tampere, Finland (R12122). 


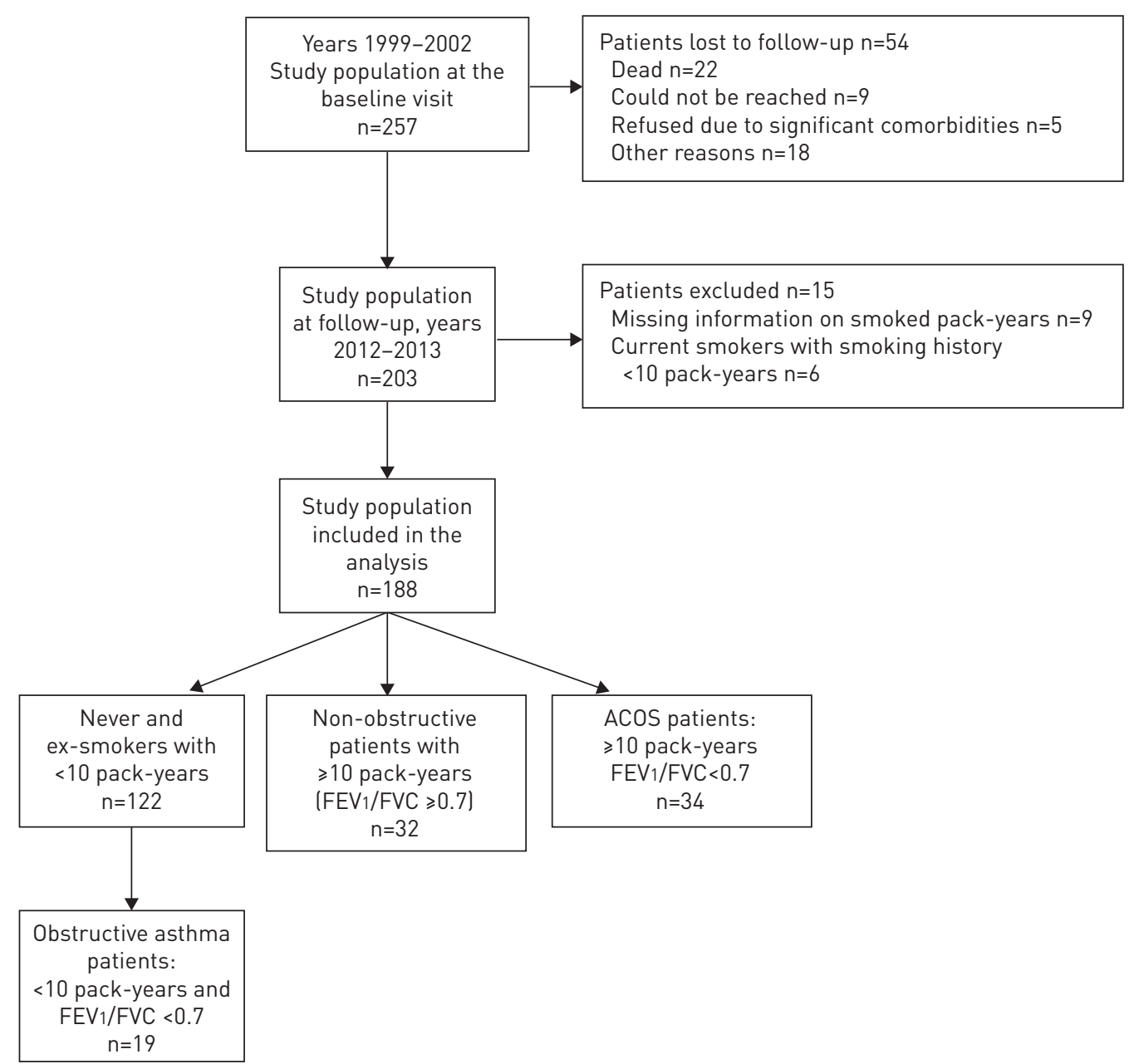

FIGURE 1 Study profile. FEV1: forced expiratory volume in 1 s; FVC: forced vital capacity.

\section{Evaluation of smoking and lung function}

Lung function measurements were performed with a spirometer (Vmax Encore 22, Viasys Healthcare, Palm Springs, CA, USA) that was calibrated daily. Finnish lung function reference values were used [24]. Lifelong cumulative exposure to tobacco was evaluated by assessing smoked pack-years (20 cigarettes per day for 1 year), and patients were divided into three groups based on smoked pack-years and lung function as follows: 1) never- and ex-smokers with $<10$ pack-years of smoking (current smokers excluded); 2) non-obstructive patients with $\geqslant 10$ pack-years and post-bronchodilator (BD) forced expiratory volume in 1 $\mathrm{s}(\mathrm{FEV} 1)$ /forced vital capacity $(\mathrm{FVC}) \geqslant 0.7$; and 3$)$ ACOS patients i.e. $\geqslant 10$ pack-years of smoking and post $\mathrm{BD}$ $\mathrm{FEV} 1 / \mathrm{FVC}<0.7$ (figure 1). The differences between obstructive asthma (with $<10$ pack-years) and ACOS were also analysed. Patients with obstructive asthma were separated from the group of never- and ex-smokers with $<10$ pack-years based on post $\mathrm{BD}$ FEV1/FVC; thus, patients with post BD FEV1/FVC $<0.7$ were categorised as obstructive asthma patients ( $\mathrm{n}=19)$ (figure 1). Serum interleukin-6 (IL-6), high-sensitivity C-reactive protein (hsCRP), immunoglobulin E (IgE), blood cell counts and fractions of exhaled nitric oxide (FeNO) were measured as previously described [25-27], and patients completed clinical questionnaires of the Asthma Control Test (ACT), COPD Assessment Test (CAT) and Asthma Questionnaire 20 (AQ20) [28] during the visit. Asthma control was evaluated based on the recommendations of the GINA 2010 report [29].

\section{Statistical analyses}

Continuous data were expressed as mean $\pm \mathrm{SD}$ or median and interquartile range, as required. Groups were compared using the t-test, Mann-Whitney rank sum test or Chi-squared test. Comparisons between three groups were done by one-way ANOVA with Tukey's post hoc test, Kruskal-Wallis test or Chi-squared test. Statistical analyses were performed using the SPSS software, version 24 (IBM SPSS, Armonk, NY, USA). A p-value $<0.05$ was regarded as statistically significant. 


\section{Results}

Out of the 188 patients analysed, 34 patients (18.1\%) were classified as having ACOS and 32 patients $(17.0 \%)$ belonged to the group of non-obstructive patients with $\geqslant 10$ pack-years. The mean \pm SD age of asthma onset in the entire cohort was $46.5 \pm 13.6$ years, and in the ACOS group, it was $53.0 \pm 10.8$ years. The majority of patients $(122 ; 64.9 \%)$ were never- or ex-smokers with a smoking history less than 10 pack-years. ACOS patients were generally older as compared to the other groups, and a male predominance was observed in the two groups with a smoking history of $\geqslant 10$ pack-years. The duration of asthma was equal among all groups, owing to the 12-year follow-up period in each. Characteristics of the three groups are presented in table 1, and those of the excluded patients are presented in table S2.

The number of patients with uncontrolled asthma (55.9\%) in the ACOS group was higher than that of the other groups. In addition, the percentage of patients with well-controlled asthma was lower in the two groups with a smoking history $\geqslant 10$ pack-years (table 1). Surprisingly, the use of oral steroids showed no difference among any of the groups, and equal percentages of patients among all groups were using inhaled glucocorticoids daily. However, the group of non-obstructive patients with a smoking history $\geqslant 10$ pack-years used higher doses of inhaled glucocorticoids and more frequently had long-acting beta-agonists in daily use. Daily use of LAMA, leukotriene antagonists or theophylline was similar among the groups (table 1). Prevalence of rhinitis, atopy and allergies showed no significant differences among groups (table 1).

We assessed whether ACOS differs from asthma, by using questionnaires that are widely validated for clinical use. The results revealed no significant differences in ACT scores or CAT scores between ACOS and non-obstructive patients with a history of $\geqslant 10$ pack-years, although ACT scores were generally lower, and CAT scores generally higher in the two groups with a smoking history $\geqslant 10$ pack-years, as compared

TABLE 1 Characteristics of the study groups

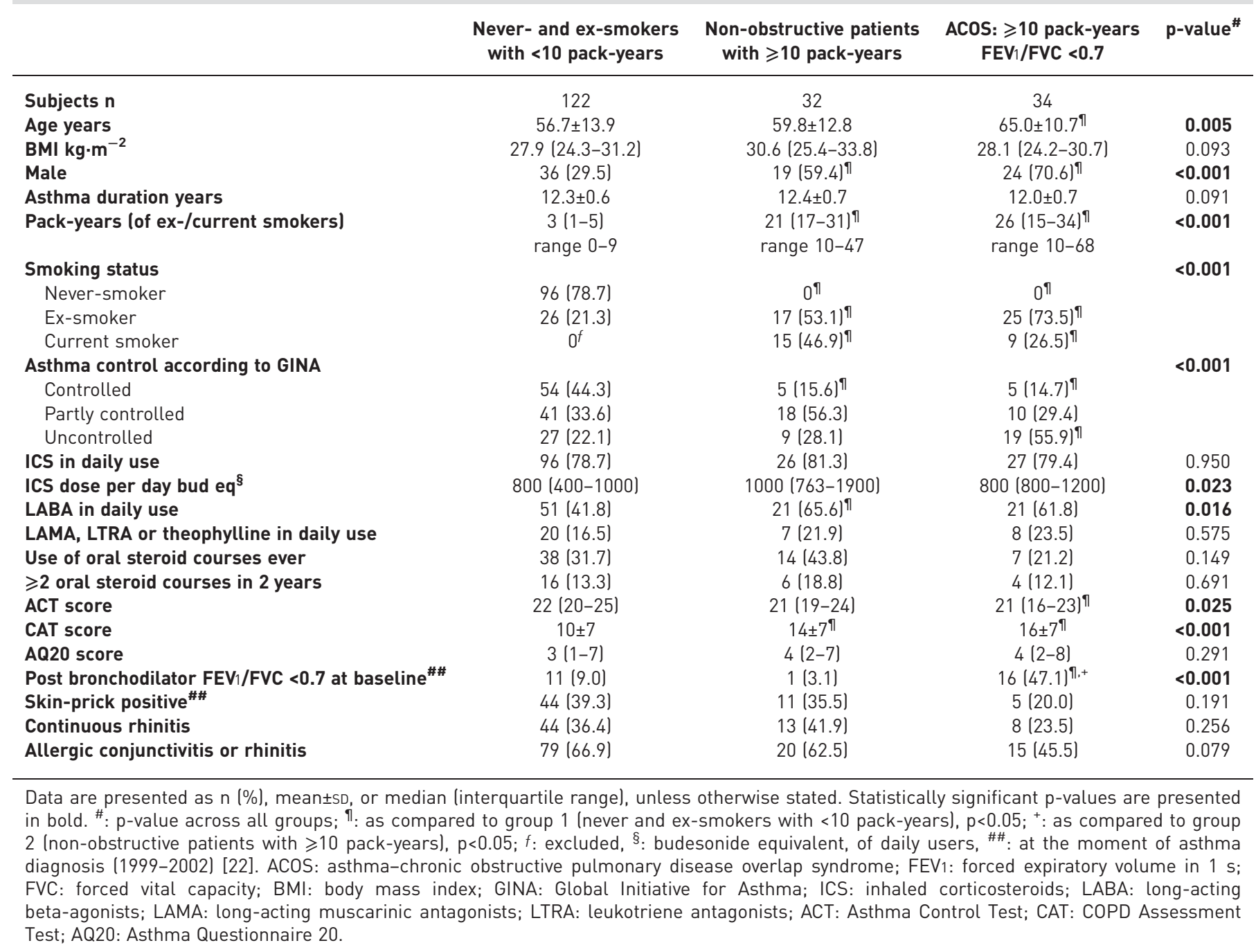


to never- or ex-smokers with $<10$ pack-years of smoking (table 1). The AQ20 scores showed no significant differences among groups (table 1).

\section{Diffusing capacity and biomarkers}

ACOS patients had significantly lower diffusing capacity of the lungs for carbon monoxide (DLCO \% and $\mathrm{DLCO} / V_{\mathrm{A}} \%$ predicted) as compared to the other groups $(\mathrm{p}=0.001)$. Furthermore, the blood neutrophil count and serum IL-6 levels were found to be the highest in the ACOS group (table 2). In contrast, the levels of blood eosinophils, hsCRP, IgE, and FeNO showed no significant differences among any of the groups (table 2).

\section{Lung function}

Post BD spirometry values of FEV1 $(\mathrm{p}=0.002), \mathrm{FEV}_{1} \%$ predicted $(\mathrm{p}<0.001)$, and FEV1/FVC ratio $(\mathrm{p}<0.001)$ were found to be significantly lower in the group of patients with ACOS as compared to those of the other groups. It should be noted, however, that post $\mathrm{BD}$ FEV1/FVC $<0.7$ was an inclusion criteria for the ACOS group. The FVC ( $\mathrm{L}$ or \% predicted) values showed no significant differences among groups (table 3). Pre-BD values are presented in table S3. We also evaluated reversibility (measurements before and after BD) of the airways at the follow-up visit, by which patients had been actively treated for asthma for 12 years. There were significantly higher levels of reversibility of the airways among patients with ACOS as compared to those of other groups. This was evident in both the FEV1 \% predicted, and the FVC $\mathrm{mL}$ and \% predicted (table 3 ).

\section{Comorbidities}

The overall number of comorbidities was significantly higher in the ACOS group as compared to the other groups $(p=0.008)$. In the ACOS group, COPD was not considered a comorbidity. Similarly, the number of medications used for the treatment of comorbidities was highest in the ACOS group. The prevalence of hypertension $(p=0.029)$, coronary heart disease $(p=0.012)$ and hypercholesterolaemia $(p=0.023)$ was highest in ACOS group (table 4). In contrast, no significant differences were noted in the prevalence of diabetes, systemic rheumatoid disease, or thyroid disease. In the group of non-obstructive patients with a smoking history $\geqslant 10$ pack-years, the prevalence of obesity was higher as compared to the other groups. Moreover, the prevalence of obesity among ACOS patients was similar to that among never- or ex-smokers with $<10$ pack-years (table 4 ). In addition, no significant differences were noted in the use of antipsychotic or antidepressant medication, nor therapy for dyspepsia or pain among any of the groups (data not shown).

\section{Differences between obstructive asthma and ACOS}

Patients with obstructive asthma (FEV1/FVC $<0.7$ but smoking history less than 10 pack-years) had significantly higher diffusing capacity values of the lung as compared to ACOS patients. The blood neutrophil count and serum IL-6 levels were higher in the ACOS group, as compared to the group of patients with obstructive asthma. No significant differences were found in the levels of eosinophils, IgE, hsCRP and FeNO (table 5). The CAT scores in the ACOS group were higher than in the group of patients with obstructive asthma; however, no significant differences were found in the AQ20 or ACT scores (table S4). Furthermore, the use of medication was similar among patients with ACOS and those with obstructive asthma, and no

TABLE 2 Diffusing capacity and biomarker data in study groups

\begin{tabular}{|c|c|c|c|c|}
\hline & $\begin{array}{l}\text { Never and ex-smokers } \\
\text { with < } 10 \text { pack-years }\end{array}$ & $\begin{array}{l}\text { Non-obstructive patients } \\
\text { with } \geqslant 10 \text { pack-years }\end{array}$ & $\begin{array}{c}\text { ACOS: } \geqslant 10 \text { pack-years } \\
\text { FEV } 1 / \text { FVC }<0.7\end{array}$ & p-value \\
\hline Subjects $\mathrm{n}$ & 122 & 32 & 34 & \\
\hline$D$ Lco/VA \% pred & $98 \pm 13$ & $96 \pm 18$ & $86 \pm 22^{1,+}$ & $<0.001$ \\
\hline Blood neutrophils $\times 10^{9} \mathrm{~L}^{-1}$ & $3.60(2.70-4.60)$ & $3.85(2.95-4.98)$ & $4.50(3.50-5.53)^{\pi}$ & 0.008 \\
\hline Blood eosinophils $\times 10^{9} \mathrm{~L}^{-1}$ & $0.16(0.09-0.28)$ & $0.14(0.09-0.22)$ & $0.19(0.10-0.29)$ & 0.409 \\
\hline hsCRP $\mathrm{mg} \cdot \mathrm{L}^{-1}$ & $1.24(0.56-2.33)$ & $1.18(0.74-5.02)$ & $0.93(0.59-3.04)$ & 0.369 \\
\hline $\mathrm{IL}-6 \mathrm{pg} \cdot \mathrm{mL}^{-1}$ & $1.52(1.12-2.48)$ & $2.10(1.09-5.69)$ & $2.88(1.88-4.99)^{9}$ & $<0.001$ \\
\hline \multicolumn{5}{|c|}{ 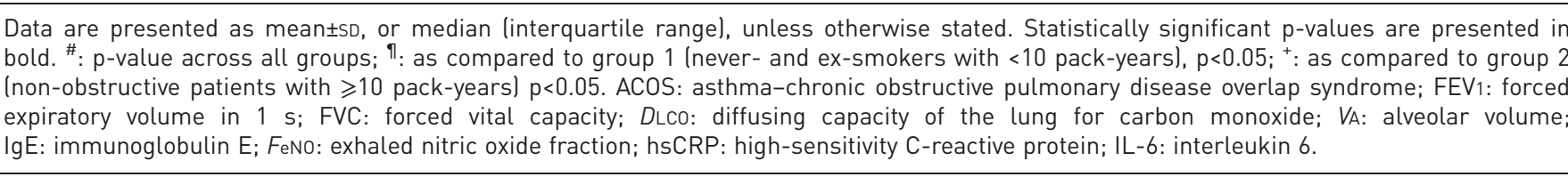 } \\
\hline
\end{tabular}


TABLE 3 Lung function in study groups

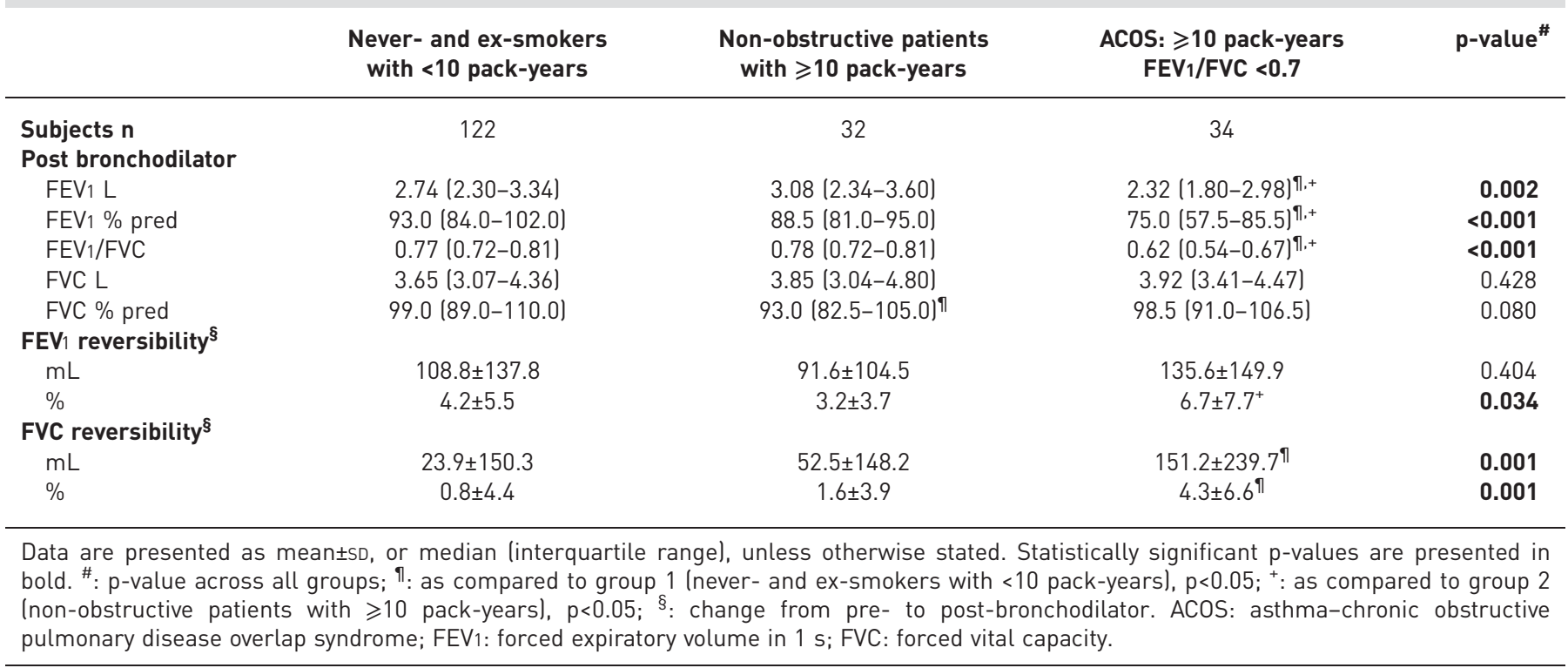

significant differences in lung function were observed (tables S4, S5). ACOS patients had a higher number of comorbidities, although no other differences were noted with respect to specific comorbidities (table S6).

\section{Discussion}

In this study, we evaluated the differences between ACOS and adult-onset asthma. ACOS differs from asthma most clearly by the lower levels of pulmonary diffusing capacity and higher levels of blood neutrophils and serum IL-6. ACOS patients have reduced lung function and higher reversibility of the airways as compared to those with asthma, despite the similar use of medication. ACOS patients also have a greater number of comorbidities than asthma patients without COPD. Furthermore, asthma control is significantly worse among ACOS patients, as compared to patients with asthma alone. To the best of our knowledge, this is the first study to evaluate both blood biomarkers and clinical characteristics that separate ACOS from asthma, in a cohort of clinical asthma patients, including also subjects with smoking-related ACOS.

Lower levels of pulmonary diffusing capacity among smokers have been considered an indicator of emphysema, a characteristic of COPD. However, little is known about the diffusing capacity among ACOS patients. In the present study, the diffusing capacity values of ACOS patients were found to be significantly lower than those of asthma patients without COPD. This is supported by the findings of KITAGUCHI et al. [30], who reported lower values of DLCO and DLCO/VA (\% predicted) among COPD patients with symptoms of asthma (defined as ACOS), as compared to those with asthma and fixed airflow limitations. Our results further

TABLE 4 Comorbidities in study groups

\begin{tabular}{|c|c|c|c|c|}
\hline & $\begin{array}{l}\text { Never and ex-smokers } \\
\text { with < } 10 \text { pack-years }\end{array}$ & $\begin{array}{l}\text { Non-obstructive patients } \\
\text { with } \geqslant 10 \text { pack-years }\end{array}$ & $\begin{array}{c}\text { ACOS: } \geqslant 10 \text { pack-years } \\
\text { FEV } 1 / \text { FVC }<0.7\end{array}$ & p-value ${ }^{\#}$ \\
\hline Subjects n & 122 & 32 & 34 & \\
\hline Number of comorbidities & $1(0-2)$ & $1(0-3)$ & $2(1-3)^{9}$ & 0.008 \\
\hline Obesity & 37 (30.3) & $19(59.4)^{9}$ & $10(29.4)^{+}$ & 0.007 \\
\hline Hypertension & $35(28.7)$ & $10(31.3)$ & $18(52.9)^{\pi}$ & 0.029 \\
\hline Coronary heart disease & $7(5.7)$ & $6(18.8)$ & $7(20.6)^{9}$ & 0.012 \\
\hline Hypercholesterolaemia & $18(14.8)$ & $8(25.0)$ & $12(35.3)^{9}$ & 0.023 \\
\hline Diabetes & 15 (12.3) & $5(15.6)$ & $8(23.5)$ & 0.264 \\
\hline Systemic rheumatoid disease & $4(3.3)$ & $1(3.1)$ & $1(2.9)$ & 0.995 \\
\hline Thyroid disease & $9(7.4)$ & $3(9.4)$ & $4(11.8)$ & 0.707 \\
\hline Number of other medications $f$ & $1(0-3)$ & $2(1-5)$ & $3(1-7)^{\pi}$ & 0.004 \\
\hline \multicolumn{5}{|c|}{$\begin{array}{l}\text { Data are presented as } \mathrm{n}(\%), \text { or median (interquartile range), unless otherwise stated. Statistically significant } \mathrm{p} \text {-values are presented in bold. } \\
\# \text { : } \mathrm{p} \text {-value across all groups; }{ }^{\uparrow}: \text { as compared to group } 1 \text { (never and ex-smokers with }<10 \text { pack-years), } p<0.05 ;{ }^{+}: \text {as compared to group } 2 \\
\text { (non-obstructive patients with } \geqslant 10 \text { pack-years), } p<0.05 ;{ }^{\S} \text { : body mass index } \geqslant 30 \mathrm{~kg} \cdot \mathrm{m}^{-2} ; f^{\prime} \text { other than medications for asthma or allergy. ACOS: } \\
\text { asthma-chronic obstructive pulmonary disease overlap syndrome; FEV1: forced expiratory volume in } 1 \mathrm{~s} ; \text { FVC: forced vital capacity. }\end{array}$} \\
\hline
\end{tabular}


TABLE 5 Diffusing capacity and biomarkers in the obstructive asthma and asthma-COPD overlap syndrome (ACOS) groups

\begin{tabular}{|c|c|c|c|}
\hline & $\begin{array}{c}\text { Obstructive asthma } \\
<10 \text { pack-years and FEV } 1 / \text { FVC }<0.7\end{array}$ & $\begin{array}{c}\text { ACOS: } \geqslant 10 \text { pack-years } \\
\text { FEV } 1 / \text { FVC }<0.7\end{array}$ & p-value \\
\hline Subjects $\mathrm{n}$ & 19 & 34 & \\
\hline DLco \% pred & $103 \pm 24$ & $85 \pm 23$ & 0.011 \\
\hline$D$ Lco $/ V_{A} \%$ pred & $100 \pm 17$ & $86 \pm 22$ & 0.018 \\
\hline Blood neutrophils $\times 10^{9} \mathrm{~L}^{-1}$ & $3.68 \pm 1.33$ & $4.54 \pm 1.37$ & 0.033 \\
\hline Blood eosinophils $\times 10^{9} \mathrm{~L}^{-1}$ & $0.16(0.08-0.20)$ & $0.19(0.10-0.29)$ & 0.217 \\
\hline $\operatorname{lgE~kU} \cdot \mathrm{L}^{-1}$ & 77 (29-198) & $59(20-140)$ & 0.399 \\
\hline FenO ppb & $11(5-24)$ & $10(5-15)$ & 0.524 \\
\hline hsCRP mg. $\mathrm{L}^{-1}$ & $0.94(0.49-1.57)$ & $0.93(0.59-3.04)$ & 0.475 \\
\hline $\mathrm{IL}-6 \mathrm{pg} \cdot \mathrm{mL}^{-1}$ & $1.64(1.12-2.21)$ & $2.88(1.88-4.99)$ & 0.001 \\
\hline
\end{tabular}

Data are shown as mean $\pm \mathrm{SD}$, or median (interquartile range), unless otherwise stated. Statistically significant $p$-values are presented in bold. FEV1: forced expiratory volume in $1 \mathrm{~s}$; FVC: forced vital capacity; $D$ LCO: diffusing capacity of the lung for carbon monoxide; $V A$ : alveolar volume; IgE: immunoglobulin $\mathrm{E} ; F_{\mathrm{eNO}}$ exhaled nitric oxide; hsCRP: high-sensitivity C-reactive protein; IL-6: interleukin 6.

suggest that measurement of the diffusing capacity could be considered a useful tool with clinical applications, when differentiating ACOS patients from those with asthma alone. In addition, a lower diffusing capacity among ACOS patients might contribute to the increased disease burden and lower quality of life suggested by the present study and previous studies [14-17]. Patients with ACOS had significantly lower lung function as compared to patients with asthma alone, as measured by the FEV1 ( $\mathrm{mL}$ and \% predicted) and FEV1/FVC ratio. This finding is consistent with those of previous studies [14-16, 31], and is feasible, as a FEV1/FVC $<0.7$ was an inclusion criterion for the ACOS group in the present study. Furthermore, our study shows that the reversibility of the airways was significantly higher in the ACOS group as compared to groups with asthma alone, at a point when the patients had been already treated for asthma for 12 years. These findings are supported by those of KITAGUCH et al. [30], who reported a greater increase in FEV1 after the bronchodilator test in the ACOS group (i.e. those with COPD and asthmatic symptoms), as compared to the group of asthma patients with airflow limitation. Our finding of higher levels of remaining reversibility in ACOS patients, who were similarly medicated for asthma, further suggests the involvement of steroid resistance [32] in ACOS.

In the present study, we found the blood neutrophil count to be significantly higher in ACOS patients than in patients with asthma. Previous studies have reported higher levels of sputum neutrophils in patients with ACOS $[33,34]$. Given the fact that inhaled glucocorticoids are known to inhibit apoptosis of neutrophils $[35,36]$, the possibility of iatrogenic neutrophilia in ACOS exists. However, in the present study, the dosages of daily inhaled glucocorticoids were not any higher in the ACOS group, in which neutrophil levels were the highest, suggesting that blood neutrophilia among ACOS patients might be derived from the actual inflammatory pathway rather than the purely iatrogenic result of the use of glucocorticoids. For example, it has been previously suggested that IL-6, being higher in ACOS patients, might promote neutrophilic inflammation in asthma [25].

Among the obstructive airway diseases, systemic inflammation has previously been typically associated with COPD. However, more recently, a similar prevalence of systemic inflammation has been reported among patients with ACOS [33]. The most widely studied biomarkers of systemic inflammation have been IL-6 and CRP [37], with which elevated levels of IL-6 and less favourable outcomes of asthma are associated [25]. We evaluated whether the assessment of blood biomarkers would help to distinguish ACOS from asthma. Our results revealed significantly higher levels of IL-6 in ACOS patients as compared to patients with asthma. This finding is supported by similar results of Fu et al. [33], whose definition of ACOS was however, not based on smoking history. Another recent study showed significantly higher concentrations of sputum IL-6 in ACOS, as compared to asthma [34]. Systemic inflammation in ACOS, including elevated CRP levels, has been proposed to resemble that in COPD [38]. In our study, the levels of hsCRP showed no significant difference among groups; an unexpected finding, considering the existence of systemic inflammation in COPD and ACOS. However, Fu et al. [33] reported similar findings in their study, and reported no significant differences in CRP levels between the ACOS and asthma groups. Thus, the results of the present study suggest that IL-6, but not hsCRP, distinguishes ACOS from asthma.

The prevalence of ACOS among patients with asthma in the present study was $18.1 \%$, which is consistent with the results of previous studies [9,16]. Over half of the ACOS patients had uncontrolled asthma, representing a significantly higher proportion of patients than in any other group. However, asthma 
control was assessed according to the GINA 2010 report [29]; thus, impaired lung function might partially explain the poor control of asthma in the present study. Previously, it has been suggested that ACOS patients might have higher CAT scores than patients with asthma [39]. Therefore, we evaluated whether ACOS can be distinguished from asthma, using questionnaires that have been validated for clinical use. We found that ACT scores, CAT scores and AQ20 questionnaires could not distinguish ACOS from asthma, even though CAT scores were higher and ACT scores were lower among patients with a history of heavier smoking ( $\geqslant 10$ pack-years). Thus, ACT, CAT and AQ20 questionnaires might not be useful in the diagnosis of ACOS among patients with asthma in clinical practice.

Moreover, our results showed a higher number of comorbidities among ACOS patients as compared to patients with asthma. Cardiovascular morbidity in particular, was found to be higher, as the prevalence of hypertension and coronary heart disease was higher in the ACOS group. This finding is supported by those of previous studies $[15,16]$.

Fixed airflow obstruction due to asthma or COPD has been widely studied. Reports suggest that lower diffusing capacity, lower FeNO levels, higher levels of neutrophils and lower eosinophil counts are evident among patients with fixed obstruction caused by COPD, as compared to those induced by asthma [4042]. However, the differences between ACOS and obstructive asthma have been far less identified [42]. When comparing obstructive asthma (with a smoking history $<10$ pack-years) and ACOS in the present study, the results reveal that ACOS most clearly differs from obstructive asthma by a lower diffusing capacity, higher number of comorbidities, and higher levels of neutrophils and IL-6 in the blood. Levels of eosinophils, IgE, FeNO and hsCRP could not distinguish obstructive asthma from ACOS.

The present study has several strengths. In our real-life clinical cohort, the diagnosis of adult-onset asthma was made by a respiratory physician, and the diagnosis was based on typical symptoms and objective lung function measurements, showing reversibility of airway obstruction [22]. The diagnosis of ACOS among our patients with asthma was based on a significant history of smoking $(\geqslant 10$ pack-years) combined with post BD FEV1/FVC $<0.7$. The duration of asthma was equal among all groups, making it possible to compare variables reliably without bias from differences in duration of the disease. However, some limitations remain in the interpretation of our results. The number of patients in the two groups of $\geqslant 10$ pack-years were somewhat low ( $n=32$ and 34 , respectively), which might lead to a loss of power in the analyses. Thus, further clinical studies with larger study cohorts are still needed. We did not have a control group of healthy persons, which could also be considered a limitation of the study. We acknowledge that a recent consensus definition of ACOS has been published, in which a key suggested feature of ACOS should be the diagnosis of asthma or atopy before 40 years of age [10]. However, another recent study has shown that the majority of adult-onset asthma is actually diagnosed at an older age [43], which makes the proposed age limit of 40 somewhat low. The present study cohort included only patients with adult-onset asthma, and the age of onset of asthma in our cohort was on average 46.5 years, and in the ACOS group, 53.0 years. The diagnosis of asthma was based on established guidelines, taking into account typical symptoms and objective lung function measurements showing bronchial variability. Therefore, possible bias due to incorrect categorisation of ACOS was unlikely in the present study, despite the higher age of asthma onset. However, given the fact that some of the subjects with COPD may have significant reversibility of obstruction, and some subjects with a history of smoking and asthma may have only partially reversible airway obstruction, there is no exact method by which these patients could be diagnostically categorised. Therefore, we acknowledge the possibility of misclassification, although diagnoses were made by carefully following the existing guidelines.

In conclusion, ACOS most clearly differs from adult-onset asthma by lower levels of pulmonary diffusing capacity, and higher levels of blood neutrophils and serum IL-6. Patients with ACOS have reduced lung function, greater reversibility of the airways (despite equal medication for asthma), and a greater number of comorbidities than asthmatic patients without COPD. Measurements of the diffusing capacity could be considered a useful clinical tool to facilitate the identification of ACOS patients among those with asthma alone.

\section{Acknowledgements}

We are grateful to Aino Sepponen for her input with data management.

Author contributions: M. Tommola, P. Ilmarinen, L.E. Tuomisto, L. Lehtimäki and H. Kankaanranta designed the study and wrote the report with input from the other authors. M. Tommola performed the statistical analyses with help from P. Ilmarinen. O. Niemelä and J. Haanpää contributed to laboratory and clinical physiology analyses, respectively. All authors contributed to interpretation of the data. All authors made critical revisions of the manuscript and approved the final version of the manuscript.

\section{References}

1 Global Initiative for Asthma. Global Strategy for Asthma Management and Prevention. Updated 2016. www.ginasthma.org Date last accessed: November 15, 2016. 
2 Global Initiative for Chronic Obstructive Lung Disease. Global Strategy for the Diagnosis, Management, and Prevention of Chronic Obstructive Pulmonary Disease. Updated 2016. http://goldcopd.org/ Date last accessed: May 10, 2016.

3 Global Initiative for Asthma and Global Initiative for Chronic Obstructive Lung Disease. Diagnosis of Diseases of Chronic Airflow Limitation: Asthma, COPD and Asthma-COPD Overlap Syndrome (ACOS) 2015. www.ginasthma. org/ Date last accessed: May 2, 2016.

4 Kankaanranta H, Harju T, Kilpeläinen M, et al. Diagnosis and pharmacotherapy of stable chronic obstructive pulmonary disease: the Finnish guidelines. Basic Clin Pharmacol Toxicol 2015; 116: 291-307.

5 Miravitlles M, Soler-Cataluña JJ, Calle M, et al. A new approach to grading and treating COPD based on clinical phenotypes: summary of the Spanish COPD guidelines (GesEPOC). Prim Care Respir J 2013; 22: 117-121.

Postma DS, Rabe KF. The asthma-COPD overlap syndrome. N Engl J Med 2015; 373: 1241-1249.

7 Miravitlles M, Vogelmeier C, Roche N, et al. A review of national guidelines for management of COPD in Europe. Eur Respir J 2016; 47: 625-637.

8 Barrecheguren M, Esquinas C, Miravitlles M. The asthma-chronic obstructive pulmonary disease overlap syndrome (ACOS): opportunities and challenges. Curr Opin Pulm Med 2015; 21: 74-79.

9 Wurst KE, Kelly-Reif K, Bushnell GA, et al. Understanding asthma-chronic obstructive pulmonary disease overlap syndrome. Respir Med 2016; 110: 1-11.

10 Sin DD, Miravitlles M, Mannino DM, et al. What is asthma-COPD overlap syndrome? Towards a consensus definition from a round table discussion. Eur Respir J 2016; 48: 664-673.

11 De Marco R, Pesce G, Marcon A, et al. The coexistence of asthma and chronic obstructive pulmonary disease (COPD): prevalence and risk factors in young, middle-aged and elderly people from the general population. PLoS One 2013; 8: e62985.

12 Miravitlles M, Soriano JB, Ancochea J, et al. Characterisation of the overlap COPD-asthma phenotype. Focus on physical activity and health status. Respir Med 2013; 107: 1053-1060.

13 Hardin M, Cho M, McDonald ML, et al. The clinical and genetic features of the COPD asthma overlap syndrome. Eur Respir J 2014; 44: 341-350.

14 Menezes AMB, Montes de Oca M, Pérez-Padilla R, et al. Increased risk of exacerbation and hospitalization in subjects with an overlap phenotype COPD-asthma. Chest 2014; 145: 297-304.

15 Milanese M, Di Marco F, Corsico AG, et al. Asthma control in elderly asthmatics. An Italian observational study. Respir Med 2014; 108: 1091-1099.

16 Kauppi P, Kupiainen H, Lindqvist A, et al. Overlap syndrome of asthma and COPD predicts low quality of life. J Asthma 2011; 48: 279-285.

17 Andersén $\mathrm{H}$, Lampela P, Nevanlinna A, et al. High hospital burden in overlap syndrome of asthma and COPD. Clin Respir J 2013; 7: 342-346.

18 Sorino C, Pedone C, Scichilone N. Fifteen-year mortality of patients with asthma-COPD overlap syndrome. Eur J Intern Med 2016; 34: 72-77.

19 Lange P, Colak Y, Ingebrigtsen TS, et al. Long-term prognosis of asthma, chronic obstructive pulmonary disease, and asthma-chronic obstructive pulmonary disease overlap in the Copenhagen City Heart study: a prospective population-based analysis. Lancet Respir Med 2016; 4: 454-462.

20 Kostikas K, Clemens A, Patalano F. The asthma-COPD overlap syndrome: do we really need another syndrome in the already complex matrix of airway disease? Int J Chron Obstruct Pulmon Dis 2016; 11: 1297-1306.

21 Tho NV, Park HY, Nakano Y. Asthma-COPD overlap syndrome (ACOS): A diagnostic challenge. Respirology 2016; 21: 410-418.

22 Kankaanranta H, Ilmarinen P, Kankaanranta T, et al. Seinäjoki adult asthma study (SAAS): a protocol for a 12 -year real-life follow-up study of new-onset asthma diagnosed at adult age and treated in primary and specialised care. NPJ Prim Care Respir Med 2015; 25: 15042.

23 Haahtela T, Klaukka T, Koskela K, et al. Asthma programme in Finland: a community problem needs community solutions. Thorax 2001; 56: 806-814.

24 Viljanen AA, Halttunen PK, Kreus KE, et al. Spirometric studies in non-smoking, healthy adults. Scand J Clin Lab Invest Suppl 1982; 159: 5-20.

25 Ilmarinen P, Tuomisto LE, Niemelä O, et al. Comorbidities and elevated IL-6 associate with negative outcome in adult-onset asthma. Eur Respir J 2016; 48: 1052-1062.

26 Tuomisto LE, Ilmarinen P, Niemelä O, et al. A 12-year prognosis of adult-onset asthma: Seinäjoki Adult Asthma Study. Respir Med 2016; 117: 223-229.

27 Tommola M, Ilmarinen P, Tuomisto LE, et al. The effect of smoking on lung function: a clinical study of adult-onset asthma. Eur Respir J 2016; 48: 1298-1306.

28 Barley EA, Quirk FH, Jones PW. Asthma health status measurement in clinical practice: validity of a new short and simple instrument. Respir Med 1998; 92: 1207-1214.

29 Global Initiative for Asthma: Global Strategy for Asthma Management and Prevention. 2010. www.ginasthma.org Date last accessed: March 29, 2011.

30 Kitaguchi Y, Yasuo M, Hanaoka M. Comparison of pulmonary function in patients with COPD, asthma-COPD overlap syndrome, and asthma with airflow limitation. Int J Chron Obstruct Pulmon Dis 2016; 11: 991-997.

31 DeMarco R, Marcon A, Rossi A, et al. Asthma, COPD and overlap syndrome: a longitudinal study in young European adults. Eur Respir J 2015; 46: 587-590.

32 Barnes PJ. Corticosteroid resistance in patients with asthma and chronic obstructive pulmonary disease. J Allergy Clin Immunol 2013; 131: 636-645.

33 Fu JJ, McDonald VM, Gibson PG, et al. Systemic inflammation in older adults with asthma-COPD overlap syndrome. Allergy Asthma Immunol Res 2014; 6: 316-324.

34 Gao J, Iwamoto H, Koskela J, et al. Characterization of sputum biomarkers for asthma-COPD overlap syndrome. Int J Chron Obstruct Pulmon Dis 2016; 11: 2457-2465.

35 Zhang X, Moilanen E, Kankaanranta $\mathrm{H}$. Beclomethasone, budesonide and fluticasone propionate inhibit human neutrophil apoptosis. Eur J Pharmacol 2001; 431: 365-371.

36 Zhang X, Moilanen E, Adcock IM, et al. Divergent effect of mometasone on human eosinophil and neutrophil apoptosis. Life Sci 2002; 71: 1523-1534. 
37 Paone G, Leone V, Conti V, et al. Blood and sputum biomarkers in COPD and asthma: a review. Eur Rev Med Pharmacol Sci 2016; 20: 698-708.

38 Gibson PG, McDonald VM. Asthma-COPD overlap 2015: now we are six. Thorax 2015; 70: 683-691.

39 Kurashima $\mathrm{K}$, Takaku Y, Ohta C, et al. COPD assessment test and severity of airflow limitation in patients with asthma, COPD, and asthma-COPD overlap syndrome. Int J Chron Obstruct Pulmon Dis 2016; 11: 479-487.

40 Fabbri LM, Romagnoli M, Corbetta L, et al. Differences in airway inflammation in patients with fixed airflow obstruction due to asthma or chronic obstructive pulmonary disease. Am J Respir Crit Care Med 2003; 167: 418-424.

41 Contoli M, Baraldo S, Marku B, et al. Fixed airflow obstruction due to asthma or chronic obstructive pulmonary disease: 5-year follow-up. J Allergy Clin Immunol 2010; 125: 830-837.

42 Rogliani P, Ora J, Puxeddu E, et al. Airflow obstruction: is it asthma or is it COPD? Int J Chron Obstruct Pulmon Dis 2016; 11: 3007-3013.

43 Kankaanranta H, Tuomisto LE, Ilmarinen P. Age-specific incidence of new asthma diagnoses in Finland. J Allergy Clin Immunol Pract 2017; 5: 189-191. 